\title{
Reformulation et acquisition de la complexité linguistique*
}

\author{
Claire MARTINOT ${ }^{1}$
}

\section{Simplification, complexification et reformulation}

Acquérir sa langue maternelle implique non seulement d'apprendre à construire des séquences de un ou plusieurs mots exprimant une signification, ce sur quoi il y a consensus dans les recherches en acquisition, mais aussi, et surtout, d'apprendre à complexifier ses énoncés. Nous faisons l'hypothèse que le petit enfant produit d'abord une prédication (i) de sens $\mathrm{X}$ puis quelque temps plus tard une prédication (ii) de sens $\mathrm{X}$ et encore plus tard une prédication (iii) de sens X. Autrement dit, l'acquisition de la langue se réaliserait en grande partie à travers l'acquisition de la compétence paraphrastique (Martinot, sous presse) et l'acquisition de la compétence paraphrastique serait le moyen privilégié pour accroître le degré de complexité de la langue, propriété partagée par toutes les langues naturelles pratiquées par les locuteurs adultes. L'objectif de cet article est de décrire et de classer les différentes procédures de complexification que des enfants de 4 à 10 ans mettent en ouvre au cours d'une expérimentation qui permet d'observer la mise en place de ces procédures.

L'acquisition de la compétence paraphrastique ne commence pas avant 4 ans, auparavant, l'enfant doit apprendre, le plus souvent dans l'interaction avec sa mère, à répéter une prédication simple puis à introduire une variation (Tomasello, $2003: 173)^{2}$. Ces trois modes fondamentaux de construction des premiers discours, la répétition, le changement (variation qui modifie le sens) et la paraphrase (variation qui ne modifie pas le sens) obéissent à un même principe explicatif que nous avons défini (Martinot, 1994, 2007 : 183) par le concept de reformulation.

Nous montrerons ici quelles procédures de reformulation permettent d'expliquer le double phénomène acquisitionnel, non élucidé à ce jour :

1. le phénomène de simplification par l'enfant des énoncés produits par les adultes, phénomène qui a lieu dans une première phase de l'acquisition, en gros jusqu'à 6-7 ans,

\footnotetext{
* Université Paris Descartes; MoDyCo, UMR 7114

49, avenue de Condé - F- 94100 - Saint-Maur-des-Fossés

cmartinot@free.fr
} 
2. le phénomène de complexification par l'enfant de ses énoncés antérieurs, phénomène qui a lieu dans une seconde phase, en gros à partir de 6-7 ans. Parmi les différentes manifestations de complexification linguistique, nous analyserons en particulier les différents types de paraphrases mis en œuvre par les enfants.

\subsection{Définition de la reformulation}

Nous définissons la reformulation comme tout processus de reprise d'un énoncé antérieur qui maintient, dans l'énoncé reformulé, une partie invariante à laquelle s'articule le reste de l'énoncé, partie variante par rapport à l'énoncé source (Martinot, 1994). D’un point de vue fonctionnel, la reformulation est un principe dynamique de production du discours dont la finalité est la construction du sens. Un discours est toute chose qui peut être dite ou écrite ${ }^{3}$. Le principe de la reformulation, simple pour tout esprit humain capable de reconnaître entre deux énoncés linguistiques ce qui est semblable et ce qui est différent, est fondé sur une combinaison entre une partie invariante que le locuteur reprend à un énoncé source (ES), point de départ du processus de reformulation, et une partie variante, nouvelle, que le locuteur articule à la partie invariante pour produire un énoncé reformulé (ER), aboutissement du processus de reformulation. Ce principe est toujours présent dans toute reformulation, que l'énoncé reformulé maintienne ou non le sens de l'énoncé source (pour une typologie des reformulations voir Martinot et al., 2008 : 222). Dans la mise en relation d'un énoncé source et d'un énoncé reformulé, les enfants s'approprient progressivement le fonctionnement de leur langue maternelle au cours d'un double mouvement qui permet à l'enfant de maintenir quelque chose de l'énoncé source et de modifier quelque chose dans son énoncé reformulé par rapport à l'énoncé source. Comme nous l'avons vu plus haut, l'enfant a le choix entre trois postures linguistiques différentes. Premièrement, il peut répéter à l'identique ou presque l'énoncé source dans une posture de répétition :

[1] ES. Elle tenait par la main une petite fille

ER. Pauline (4 ans). elle *tena par la main une petite fille

Deuxièmement, il peut modifier l'énoncé source en donnant un autre sens à son énoncé tout en conservant de l'énoncé source une partie invariante (ci-dessous qui savent) :

[2] ES. Je veux apprendre à parler avec les oiseaux qui savent tout ce qui se passe dans le ciel

ER. Edward (6 ans). Je voudrais apprendre à parler avec les oiseaux qui savent voler Troisièmement, il peut modifier l'énoncé source en conservant le sens de celui-ci dans son énoncé reformulé (ci-dessous, cas de paraphrase descriptive définitoire ${ }^{4}$ ) : 


\section{[3]ES. Julie sera ta voisine}

ER. Ilan (8 ans). Julie sera à côté de toi

\subsection{Simplification et complexification : deux notions à définir}

\subsubsection{Simplification des énoncés sources entendus}

$\mathrm{Au}$ cours de la première phase de l'acquisition de la langue maternelle, il faut expliquer comment les enfants parviennent à passer des énoncés qu'ils entendent aux énoncés qu'ils produisent. Autrement dit, si l'on admet - sous la pression d'une intuition forte et sans doute consensuelle - que la langue maternelle offerte à l'enfant est constituée d'énoncés majoritairement plus complexes que les énoncés simples produits par le jeune enfant - c'est le cas jusqu'à 6-7 ans (Martinot, 2005) - il faut expliquer comment celui-ci parvient à simplifier les énoncés complexes qui font sens pour lui et qu'il utilise dans sa production. Les procédures de reformulation utilisées par les enfants leur permettent de simplifier les énoncés sources de la langue cible, par exemple, au tout début de cette première phase de l'acquisition, en repérant et en reproduisant uniquement les mots nécessaires à l'expression d'une prédication :

[4] a. ES (mère). Tu veux pas faire dodo ?

ER (enfant) Pas dodo (Dans l'interaction mère-enfant, ER est la réponse de l'enfant à sa mère)

b. ES (mère). Allez viens on prend un bain.

ER (enfant). prend bain (Dans l'interaction, ER est un énoncé d'acquiescement)

Les énoncés ER ci-dessus, produits par des enfants d'un plus de 2 ans, sont incomplets grammaticalement, mais n'en expriment pas moins une prédication. Cette aptitude prédicative extrêmement précoce est universelle au même titre que l'aptitude à mettre en œuvre la grammaire de sa langue.

\subsubsection{Complexification des énoncés enfantins produits}

Il faut ensuite et surtout expliquer comment l'enfant, grosso modo à partir de 7 ans, parvient à produire des énoncés qui atteindront progressivement le degré de complexité d'un grand nombre d'énoncés adultes.

Le processus de complexification en langue suppose une hiérarchie entre des degrés de complexité et donc des critères permettant de distinguer un énoncé simple d'un énoncé complexe, ou plutôt un énoncé plus simple d'un énoncé plus complexe. En effet, la simplification comme la complexification réfèrent à un jugement linguistique relatif qui fait 
intervenir divers critères et différents niveaux propres à la langue mais aussi extérieurs à la langue.

Les deux phrases suivantes n'ont pas le même degré de complexité sémantique ou conceptuelle : le soleil brille; Dieu est. Elles sont toutes deux considérées par toutes les théories linguistiques et grammaticales comme des phrases syntaxiquement simples, des discours élémentaires (dans la terminologie de Harris) mais la seconde est assurément plus complexe à interpréter et à produire que la première. Par ailleurs, une phrase telle que les enfants entendirent un grincement est considérée comme une phrase syntaxiquement simple par la tradition grammaticale ou l'école fonctionnaliste (Tchekhoff, 1977) mais comme une phrase syntaxiquement complexe par le courant harrissien $(1976,1988)$ qui analyse cette phrase apparemment simple (un seul verbe conjugué) comme une phrase dérivée par réduction, à partir de deux phrases élémentaires : (a) les enfants entendirent quelque chose, (a') un grincement a eu lieu > (b) les enfants entendirent qu'un grincement a eu lieu > (c) les enfants entendirent un grincement. Tous les éléments supprimés en (c) sont redondants du point de vue de l'information, qui est la même en (a-a') et en (c). Pour Harris (trad. franç. 2007 : 45) «la complexité notoire de la grammaire, dont la majeure partie est créée par les réductions, n'est pas due à la complexité de l'information et n'est pas nécessaire à l'information ». Cette hypothèse de Harris est contestee par l'hypothèse cognitiviste de Givón (2009: 10): «more complex mentally represented events are coded by more complex linguistic/syntactic structures $»^{5}$. L'exemple de la phrase Dieu est donnerait plutôt raison à Harris. D’une façon plus générale, il y a consensus pour admettre que plus la syntaxe est intégrée et plus le degré de complexité est grand: juxtaposition > coordination > subordination > prédication complexe (constituée par ex. d'une prédication principale et d'un complément prépositionnel provenant de la réduction d'une proposition circonstancielle: dans sa grande fureur, il a oublié ses clefs). Il est vraisemblable que l'interprétation sémantique soit également plus complexe en fonction de la condensation ou de la synthétisation de l'information. Cela vaut pour les constructions comme pour le lexique: chuchoter est plus complexe que dire parce que chuchoter nécessite dire dans sa définition analytique : dire à voix chuchotée (Ibrahim, 2001, Ibrahim et Martinot, 2003). Un autre facteur de complexité, difficile à mesurer, provient de la fréquence relative d'un terme ou d'une construction entendus par un locuteur donné et en particulier par un enfant (Tomasello, 2003 : 173). On peut cependant considérer que la fréquence n'est que la conséquence d'un phénomène linguistique que Harris a fort bien décrit (pour un dernier état de la formulation, cf. 1988, trad. fr. 2007 : 37-38) : plus un mot a une contrainte de sélection forte relativement à 
un autre mot, plus ce mot sera spécifique et moins il sera fréquent : de ce point de vue, le verbe manger, par exemple, a une contrainte de sélection moins forte que déguster ou enfourner.

On peut donc s'attendre à ce que la fréquence d'occurrence et la complexité structurelle interagissent de façon complexe dans le processus développemental (ibid. 175). Ce point ne sera pas abordé dans notre propos.

Le point de vue harrissien présente un immense avantage dans le domaine de la linguistique de l'acquisition : il permet de rendre compte du fait que jusqu'à 6-7 ans la quasi-totalité des phrases à un verbe conjugué, produites par les enfants de façon spontanée, sont des phrases élémentaires, c'est-à-dire contenant un seul prédicat (contrairement à (c) ci-dessus qui contient deux prédicats) mais aussi des phrases dans lesquelles les noms (ou les pronoms) sont dans $90 \%$ des cas les arguments du verbe (Martinot, 2005) ${ }^{6}$. Le mode d'organisation de la langue selon la conception de Harris est une organisation hiérarchisée : certaines phrases sont élémentaires, d'autres sont dérivées donc plus complexes (ou plus marquées, selon Givón, 1995). Cette conception nous a permis de rendre compte du fait que les enfants repèrent, dès 4 ans, dans une histoire qu'ils doivent restituer, les prédications principales relativement aux prédications secondes (pour une revue de la question sur la prédication seconde, voir Furukawa, 1996 ; Cadiot et Furukawa, 2000) et que ces dernières ne sont reformulées fréquemment, qu'à partir de 8 ans (Martinot et al. 2009). On a donc pu en conclure que la reformulation des seules prédications principales correspond à un degré moindre de complexité syntaxique que la reformulation d'une prédication complexe constituée d'une prédication principale et d'une prédication seconde.

Voyons quelques exemples de reformulations (extraits du protocole expérimental que nous présenterons plus bas) qui montrent comment l'enfant simplifie d'abord un énoncé source complexe puis le reformule ensuite avec un degré croissant de complexité.

L'énoncé source :

[5) je veux apprendre à parler avec les oiseaux qui savent tout ce qui se passe dans le ciel

est d'abord reformulé à 4 ans par :

[6] je veux apprendre à parler avec les oiseaux

L'énoncé source résulte d'une double simplification : du fait de l'effacement de la relative explicative qui est une prédication seconde, et du fait de la répétition stricte de la principale. Dans l'hypothèse d'une acquisition par reformulation, que nous défendons depuis Martinot (2000), la reformulation par répétition (cf. [1] ci-dessus) est considérée comme moins difficile 
à mettre en œuvre que la reformulation par équivalence (cf. [3] ci-dessus) du fait que la répétition ne nécessite que la mémoire de l'enfant et ne dit rien sur la compréhension ou sur la re-construction du sens avec d'autres moyens linguistiques que ceux qui se trouvent dans l'énoncé source?

L'énoncé source [5] est reformulé à 6 ans par :

[7] je veux apprendre à parler avec les oiseaux pour voir tout ce qui se passe dans le ciel

La principale est encore répétée, comme à 4 ans, et la relative est reformulée par la proposition : pour voir ...ciel, qui implique que le sujet de la principale soit le même que le sujet de cette proposition en pour, ce qui modifie le sens. La coréférence du sujet est plus simple à envisager mais l'occurrence d'une reformulation formellement proche de la relative source est plus complexe que son effacement qui avait lieu à 4 ans.

Le même énoncé source est reformulé à 8 ans par :

[8] je voudrais savoir parler aux oiseaux qui savent tout ce qu'il y a dans le ciel La proposition principale et la relative sont toutes deux reformulées par une équivalence (et non plus par une répétition et un changement de sens): paraphrase fonctionnelle de la principale (apprendre à faire quelque chose a pour conséquence savoir faire cette chose, en discours les deux verbes sont équivalents); paraphrase sémantique de la relative (tout ce qui se passe dans le ciel est synonyme, même hors contexte de tout ce qu'il y a dans le ciel). Le degré de complexité linguistique est plus grand chez cet enfant de 8 ans que chez un enfant plus jeune [7] du fait de l'occurrence de deux reformulations paraphrastiques et de la reprise de la construction relative.

Et enfin, l'énoncé source [5] est reformulé à 10 ans par :

[9] je voudrais pouvoir parler aux oiseaux de tout ce qu'ils savent dans le ciel

Le degré d'intégration syntaxique est ici maximal avec la restructuration des deux propositions sources en une prédication complexe, transformation particulièrement difficile à réaliser et d'ailleurs peu répandue même chez les enfants de 10 ans, dans notre corpus.

\section{La théorie de la reformulation : une nouvelle théorie acquisitionnelle ?}

\subsection{Rôle accordé à la langue cible - l'acquisition : un processus long et continu}

Dans le débat actuel qui oppose les tenants de la Grammaire générative aux tenants de la théorie-de-l'acquisition - du-langage-fondée-sur - l'usage désormais TALFU (A UsageBased Theory of Language Acquisition), branche explicitement cognitiviste des Grammaires de construction (Tomasello, 2003 ; Lieven \& Tomasello, 2008 : 171), ces derniers estiment 
que le problème de la diversité des langues ne peut être résolu par le postulat d'un dispositif d'acquisition universel et abstrait, et que ce dispositif unique ne peut rendre compte des différentes étapes du processus acquisitionnel (Tomasello, $2003: 7)$. La critique adressée à la Grammaire Universelle porte aussi sur le peu de cas qu'elle fait des données linguistiques effectives fournies à l'enfant. Pour les tenants de la Grammaire générative "you can't get there from here » (Gleitman \& Wanner, 1982), alors que pour Tomasello (2003: 3): "Children can get from here to there », c'est-à-dire que les enfants peuvent partir des énoncés de la langue maternelle pour produire leurs énoncés. Nous partageons ce point de vue constructiviste sur le caractère formateur des données linguistiques fournies à l'enfant : nous postulons aussi que l'enfant acquiert sa langue maternelle en produisant ses énoncés par référence aux énoncés de la langue cible. Mais nous nous démarquons de la TALFU (Tomasello, 2003) du fait que l'explication que nous proposons, sous l'appellation de Théorie de la reformulation, reste dans le cadre strict de la langue, ce qui n'est pas le cas de la TALFU.

\subsection{Acquérir la langue par la langue}

L'explication que nous proposons d'une acquisition de la langue par la reformulation reste dans le cadre strict de la langue: l'enfant s'approprie la langue par la langue, postulat cohérent avec le cadre théorique harrissien dans lequel nous nous situons : «Les langues naturelles n'ont pas de métalangue qui leur soit extérieure » (Harris, trad. Française, 2007 : 30). Ce postulat appliqué à l'enfant signifie que c'est dans les énoncés qu'il entend - qui le concernent et qu'il retient - que l'enfant cherche à savoir comment il va dire ce qu'il veut dire. Les productions des enfants sont d'abord et avant tout des productions linguistiques que l'on peut parfaitement décrire, analyser, comparer, avec les outils de l'analyse linguistique susceptibles d'en rendre compte.

Par ailleurs, tant que l'on ne connaît absolument pas le lien exact entre les processus cognitifs très nombreux, très complexes et le fonctionnement des langues, il est prématuré de vouloir expliquer l'acquisition de la langue maternelle en recherchant la réponse dans des paradigmes dont on ne connaît pas le lien avec la langue.

Ainsi, il est tentant de vouloir expliquer l'acquisition de la langue par le seul processus mémoriel comme le fait Le Ny (2005: 99) : «Une bonne conception de la mémoire de travail, de la mémoire à long terme, et de leurs échanges et interactions, est absolument nécessaire pour pouvoir se former une idée exacte du fonctionnement et des structures du langage. Une autre façon de dire «fonctionnement » et «structures » est d'utiliser les termes 
équivalents de «processus » et « représentations », dont nous avons vu qu'ils constituent les deux parts principales de l'esprit. » Cette tentative d'explication, qui accorde une importance minime aux contraintes proprement linguistiques et à l'extrême diversité dans le fonctionnement des langues, échappe à la description systématique, fastidieuse, peu reconnue mais incontournable et falsifiable des faits de langue et ne peut pas expliquer le fonctionnement ou l'acquisition d'une langue. La mémoire est évidemment nécessaire dans tout processus d'apprentissage, comme la circulation sanguine ou la présence d'oxygène sont nécessaires à la vie humaine mais on voit bien que la présence nécessaire d'une aptitude ou d'un phénomène dans un processus général, l'apprentissage ou la vie, ne peut pas receler l'explication recherchée. De plus l'équivalence posée par l'auteur entre fonctionnement et processus d'une part, structures et représentations d'autre part, est en désaccord avec l'acception de ce qu'est une langue pour l'immense majorité des linguistes: le terme de structure en linguistique a un sens très précis qui concerne la syntaxe d'une phrase simple, éventuellement l'organisation de la langue ; le fonctionnement d'une langue est un objet de description qui entraîne, dans sa mise en œuvre par le locuteur, un certain nombre de processus évidemment mentaux, mais ces processus, sans doute comparables d'un être humain à l'autre, ne peuvent pas expliquer la diversité potentiellement illimitée des grammaires, ni le fait que l'on puisse donner la même information au moyen d'une phrase simple ou complexe, ni le fait qu'il existe toujours plusieurs énoncés de même degré de complexité pour exprimer des sens équivalents.

\section{Description et justification méthodologique}

Montrer que les enfants acquièrent leur langue maternelle en reformulant un certain nombre d'énoncés qui font sens pour eux est un défi très difficile à relever si l'on s'en tient à l'analyse de corpus spontanés dans lesquels les enfants racontent ou décrivent quelque chose. Dans les corpus provenant de séances où les enfants sont en interaction avec d'autres locuteurs, on a un peu plus de chances de trouver les paires : énoncé source-énoncé reformulé, mais ce type de corpus n'est recueilli qu'avec les très jeunes enfants (2-3 ans) qui en sont au stade de construire des prédications de plus en plus complètes, et pas encore au stade où ils complexifient les énoncés de leur stock mémorisé. Par ailleurs, la diversité syntaxique et sémantique des énoncés sources est réduite du fait qu'il s'agit paradoxalement d'un échange authentique entre mère et enfant (le plus souvent). De plus, on sait que la langue utilisée dans les échanges familiers est relativement stéréotypée et peu complexe aussi bien du point de vue syntaxique que lexical (Blanche-Benveniste, 1990). Le protocole (semi-) expérimental que 
nous utilisons depuis Martinot (2000) jusqu'à nos derniers travaux s'appuie sur la lecture faite individuellement à chaque enfant d'un texte de type littéraire, adapté à la compréhension d'enfants de 4 ans et encore digne d'intérêt pour les enfants de 10 ans, puis sur la restitution immédiate que chaque enfant fait de l'histoire, restitution enregistrée et transcrite ${ }^{8}$. Le texte source, Tom et Julie, d'une longueur moyenne de 500 mots (selon les langues dans lesquelles il a été traduit) contient un grand nombre de phénomènes syntaxiques et quelques phénomènes sémantiques que nous supposions complexes (cf. annexe). Cette situation, très peu naturelle, de demander à un enfant d'écouter une histoire puis de la raconter à son tour, n'a jamais posé de problème, les enfants étant toujours volontaires et prévenus du déroulement de la séance. La consigne donnée est la suivante : «Maintenant, c'est à toi de raconter la même histoire, essaie de ne rien oublier et tu peux utiliser tes mots à toi ».

L'intérêt de cette tâche est que l'enfant va devoir restituer, avec les moyens linguistiques dont il dispose, de nombreux et divers phénomènes complexes, concentrés dans un texte assez court. Il va devoir utiliser d'une façon ou d'une autre les énoncés sources de l'histoire mais également l'ensemble des énoncés dont il dispose, en particulier les énoncés équivalents fonctionnellement, et qui lui sont familiers, pour reconstruire l'histoire. L'ensemble des reformulations produites nous renseigne donc de façon très précise sur les compétences linguistiques de chaque enfant. Cette situation de restitution présente un point commun essentiel avec la situation naturelle d'acquisition : l'enfant est toujours soumis à une langue beaucoup plus complexe que celle qu'il produit lui-même, et c'est bien à partir de cette langue, beaucoup plus complexe, qu'il doit trouver les moyens de parler. Nous posons que les reformulations attestées dans cette tâche ne peuvent pas être différentes des reformulations qu'il produit naturellement.

Le deuxième intérêt de cette tâche est de permettre une comparaison du différentiel qui s'établit entre tel énoncé source et l'ensemble des énoncés reformulés par des enfants d'âges différents. Dans la vie courante, il est à peu près impossible de contrôler le ou les énoncés sources, d'autant plus quand l'enfant multiplie ses interlocuteurs, en grandissant.

L'émergence d'un différentiel dont on contrôle la source va nous permettre d'analyser comment l'enfant transforme les énoncés sources qu'il restitue : en répétant de façon plus ou moins réussie, en changeant le sens, en produisant un équivalent sémantique, qui correspond aux quatre types de paraphrases que nous avons signalés plus haut (cf. note 4) : [10] (a) sémantique, (b) fonctionnelle, [11] (c) formelle (transformation et restructuration), [12] (d) descriptive. Exemples :

[10] ES. Elle tenait par la main une petite fille que personne n'avait encore jamais vue 
(a) ER. Rémy, 8 ans. elle tenait la main d'une petite fille qu'on ne connaissait pas

(b) ER. Maxime, 10 ans. Il était une fois une petite fille qui faisait sa rentrée à l'école

[11] ES. Les enfants furent éblouis par la lumière qui inondait l'intérieur de l'arbre

(c). ER. Léo, 10 ans. à l'intérieur c'est inondé de lumière

(restructuration de la relative déterminative)

[12] ES. L'arbre était en train de tourner sur lui-même

(d). ER. Maxime, 10 ans. L'arbre fait des tours sur lui-même

Le troisième intérêt de cette tâche est qu'elle est utilisable dans n'importe quelle langue puisque c'est toujours le différentiel entre énoncé source et énoncé reformulé qui est analysé.

L'analyse du différentiel se fait prédication par prédication. Ainsi dans l'énoncé source de (ab) ci-dessus, on compare d'une part la prédication principale elle tenait par la main une petite fille et d'autre part la prédication seconde, ici une relative explicative que personne n'avait encore jamais vue avec leurs reformulations respectives. Cette façon de procéder serait inadaptée pour évaluer la maîtrise d'un récit, de sa cohérence et de sa cohésion, mais pour notre objectif qui est de repérer ici comment les enfants complexifient leurs prédications, elle est parfaitement opérationnelle, de même qu'elle nous a déjà permis de décrire dans le détail et dans différentes langues comment les enfants reformulent les relatives du texte source (Martinot et al, 2009), quelles reformulations définitoires ils produisent spontanément (Gerolimich, Martinot, De Gioia, 2009), quelles prédications sont paraphrasées (Martinot et Gerolimich, sous presse, Martinot, sous presse).

Dans cet article, nous allons tenter de montrer comment les énoncés du texte source que nous jugeons complexes sont reformulés par les enfants francophones de 4, 6, 8 et 10 ans. Pour chaque tranche d'âge, 15 enfants ont été enregistrés individuellement comme nous l'avons décrit plus haut ${ }^{9}$.

\section{Analyse des procédures de reformulation}

Selon notre hypothèse acquisitionnelle, les enfants de 4 et 6 ans de notre corpus devraient majoritairement simplifier les énoncés sources qu'ils reformulent, tandis que les enfants de 8 et 10 ans devraient majoritairement produire des reformulations dont le degré de complexité se rapproche de celui du texte source. Pour faciliter l'appariement des énoncés enfantins avec 
ceux du texte source mais aussi pour avoir une représentation le plus fidèle possible de ce que les enfants restituent ou non dans l'histoire, le texte source a été segmenté en 14 séquences narratives (cf. annexe). La quasi-totalité des 14 séquences sont restituées par la quasi-totalité des enfants de 6 à 10 ans. A 4 ans, 9 séquences sont reformulées par la quasi-totalité des enfants, les 5 autres séquences n'étant reformulées que par 4 à 9 enfants. Pour qu'une séquence source soit considérée comme reformulée, il faut qu'au moins une prédication complète de cette séquence soit présente sous une forme ou une autre dans la production de l'enfant. Cette prédication est très souvent celle qui est la plus pertinente pour la suite de l'histoire :

[13] Séq. 4. ES. Le lendemain matin, dans la cour de l'école, Tom guettait l'arrivée de sa nouvelle petite voisine. Dès qu'il l'a aperçue, il s'est dirigé vers la fillette et lui a tendu la boîte qu'il avait fabriquée pour elle, la veille.

ER. Marion, 4 ans. et le lendemain matin la donna à Julie

Nous avons sélectionné 17 prédications simples et complexes (syntaxiquement) qui correspondent à un échantillon représentatif des phénomènes lexicaux et syntaxiques supposés difficiles à restituer ou à reformuler. Les exemples qui sont donnés à chaque fois sont les reformulations les plus pertinentes pour notre propos.

Nous analyserons d'abord les prédications qui contiennent un mot lexical complexe (mot décomposable, verbes exprimant un mode d'action spécifique, prédicat nominal, construction verbale réfléchie, forme passive, verbe métaphorique, verbe opérateur) puis les prédications qui présentent a priori une difficulté d'ordre syntaxique ( 2 relatives explicatives, 1 prédication complexe comprenant une suite d'opérateurs, 1 prédication comprenant 2 compléments temporels, dont l'un seulement est pragmatiquement nécessaire, et 2 compléments locatifs pragmatiquement nécessaires).

\subsection{Complexité lexicale}

Séq. 4.1. Tom guettait l'arrivée de sa nouvelle petite voisine

Séq. 4.2. il l'a aperçue

Séq. 4.3. il s'est dirigé vers la fillette

Séq. 4.4. (il) lui a tendu la boîte

Séq. 6.1. Julie chuchota à Tom

Séq. 6.2. Tom souleva le couvercle

Séq. 6.3. (Tom) découvrit un morceau de papier

Séq. 10.1. les enfants entendirent un grincement 
Séq. 10.2. l'arbre était en train de tourner sur lui-même

Séq. 11.1. Les enfants furent éblouis par la lumière

Séq. 11.2 (la lumière) qui inondait l'intérieur de l'arbre

Séq. 12. les fleurs semblaient se parler en chantant

Nous avons écrit plus haut que l'analyse des reformulations devait se faire sur des séquences de mots correspondant à une prédication simple (par ex. $4.2,4.3 \ldots$ ) ou à une prédication complexe (par ex. 4.1, 10.1...). Les résultats que nous synthétisons ci-dessous ne concernent cependant qu'un seul mot dans chaque prédication. Il serait donc plus juste de dire que les résultats concernent le mode de restitution d'un mot dans une séquence reformulée. Par commodité, nous citerons les verbes sous leur forme infinitive.

\subsubsection{Verbes décomposables guetter, apercevoir, se diriger, chuchoter, découvrir}

Un certain nombre de verbes sont complexes du fait que leur sens peut être décomposé sous la

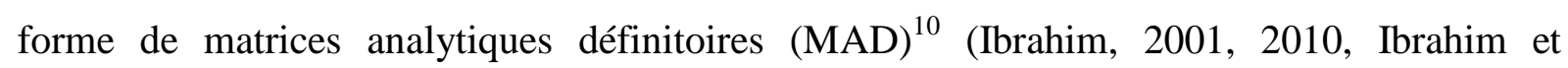
Martinot, 2004). C'est le cas des verbes guetter quelqu'un, apercevoir quelqu'un, se diriger, chuchoter quelque chose à quelqu'un, découvrir quelque chose:

Séq. 4.1. Tom guettait l'arrivée de sa nouvelle petite voisine.

MAD : Tom regardait fixement dans une direction précise en attendant Julie / Tom attendait

Julie en regardant fixement dans une direction précise

Séq. 4.2. il l'a aperçue.

MAD : il a commencé à la voir après une certaine attente

Séq. 4.3. il s'est dirigé vers la fillette

MAD : il est allé dans la direction de la fillette

Séq. 6.1. Julie chuchota à Tom

MAD : Julie dit à Tom à voix basse / chuchotée

Séq. 6.3. (Tom) découvrit un morceau de papier

MAD : Tom vit un morceau de papier alors qu'il ne s'y attendait pas

Quand les enfants utilisent respectivement regarder ou attendre pour guetter, voir pour apercevoir et découvrir, aller pour se diriger et dire pour chuchoter à l'exclusion de toute autre information, on dira qu'ils ont produit une reformulation définitoire analytique lacunaire (RDAL). Cette procédure simplifie le verbe source :

[14] Séq. 4.2. ES. Dès qu'il l'a aperçue (...) 
ER. Daphné, 6 ans. une fois qu'il la voyait (...)

[15] Séq. 4.1. ES. Tom guettait l'arrivée de sa nouvelle petite voisine

ER. Madeleine, 8 ans. il attendait Julie

Quand ils produisent ou tentent de produire une reformulation définitoire analytique complète (RDAC), qui correspond ou se rapproche des MAD ci-dessus, on considère que cette procédure est plus complexe que la précédente :

[16] Séq. 4.1. ES. Tom guettait l'arrivée de sa nouvelle petite voisine

ER. Daphné, 6 ans. il attendait * guettement sa petite voisine

ER. Océanie, 10 ans. il voit fixement la petite fille

ER. Alexandrine, 10 ans. il essaye de la voir

Le verbe guetter semble plus complexe que les autres verbes décomposables. En effet, ce n'est qu'à 10 ans que les enfants complexifient la reformulation des énoncés contenant guetter. De plus, les tentatives de reformulation définitoire analytique à 10 ans ne correspondent pas (encore) au sens complet de guetter. Remarquons que l'enfant de 6 ans (c'est le seul : [16] Daphné) a également tenté de produire une RDAC en utilisant le verbe élémentaire de la MAD et en produisant une translation à partir du verbe source : guetter > *guettement pour indiquer la manière avec laquelle Tom attendait Julie.

La production d'un synonyme du verbe source correspond à un degré de complexité sémantique (mais peut être pas fonctionnel) équivalent à celui du verbe source ${ }^{11}$ :

[17] Séq. 6.3. ES. (Tom) découvrit un morceau de papier

ER. Barbara, 10 ans. et trouva un petit papier ${ }^{12}$

[18] Séq. 6.1. ES. Julie chuchota à Tom

ER. Maxima, 8 ans. la petite fille souffla à son voisin

Dans le contexte de l'histoire, on peut admettre que souffler ait le même sens que chuchoter, dans la mesure où la MAD de souffler partage avec la MAD de chuchoter la description du procès $^{13}$. Les synonymes introduits par les enfants lors de la restitution de l'histoire correspondent à des mots immédiatement disponibles dans leur stock de phrases mémorisées d'autant que le verbe souffler dans l'ER de Maxima [18], fait partie du lexique fréquent dans les situations scolaires. La production de synonymes dans le cadre expérimental que nous utilisons nous informe des compétences lexico-syntaxiques des enfants, compétences 
alimentées par l'ensemble des discours qu'ils ont mémorisés et pas seulement par l'histoire proposée dans l'expérimentation.

A partir de 8 ans, on assiste à deux cas différents de complexification dans la mise en œuvre de la RDAL, dans lesquels l'enfant complète le verbe voir par un infinitif, provenant de la translation du déverbal l'arrivée [19]:

[19] Séq. 4.2. ES. (Tom guettait l'arrivée de sa nouvelle petite voisine). Dès qu'il l'a aperçue $(\ldots)$

ER. Ninon, 8 ans. quand il l'a vue arriver

ou bien dans lesquels l'enfant produit à la fois une analyse lacunaire du verbe source et sa répétition [20]:

[20] Séq. 6.1. ES. Julie chuchota à Tom

ER. Antonin, 8 ans. un jour chuchota et lui dit

ER. Léo, 10 ans. Julie *disa à Tom en chuchotant

L'analyse et la répétition produisent chez Antonin (8 ans) une redondance, mais plus chez Léo (10 ans) puisque dans la construction gérondive (prédication seconde qui complexifie la phrase), chuchoter n'exprime que la manière.

Une autre procédure de complexification [21], attestée à 8 et 10 ans, consiste à apporter une précision au procès verbal (paraphrase sémantique). Marc ( 8 ans) emploie une préposition inappropriée, ce qui n'est plus le cas à 10 ans (Romain). Les enfants de 4 et 6 ans ont répété le verbe chuchoter ou l'ont simplifié.

[21] Séq. 6.1. ES. Julie chuchota à Tom

ER. Marc, 8 ans. elle chuchota dans l'oreille

ER. Romain, 10 ans. elle chuchote à l'oreille

A l'exclusion de Clara (4 ans) qui produit une sorte de reformulation analytique définitoire complète [22] en décomposant non pas directement le verbe se diriger mais le verbe marcher (si l'on admet que marcher = faire des pas) et clôt le procès avec le verbe télique rejoindre, les enfants de 4 et 6 ans ont répété ou simplifié le verbe se diriger:

[22] Séq. 4.3. ES. il s'est dirigé vers la fillette

ER. Clara, 4 ans. il fit des pas et la rejoint

Dans un mouvement inverse de la procédure analytique complète ou lacunaire, certains enfants, à partir de 8 ans, synthétisent plusieurs informations, exprimées par au moins deux mots. Cette procédure est complexe, linguistiquement et cognitivement, du fait que l'enfant maintient les deux informations sources : l'idée d'immédiateté exprimée par dès que (il l'a 
aperçue) et l'idée du déplacement exprimée par se diriger et les restitue par un nouveau moyen lexical, le verbe se précipiter :

[23] Séq. 4.2 et 4.3 ES. Dès qu'il l'a aperçue, il s'est dirigé vers la fillette

ER. Marc, 8 ans. il s'est précipité sur elle

Si l'on récapitule les procédures de complexification et de simplification qui sont apparues au cours de la reformulation des cinq verbes décomposables, donc sémantiquement complexes, ici choisis, on peut dire que les procédures de reformulation par simplification, souvent exclusives à 4 et 6 ans et significativement plus fréquentes qu'à 8-10 ans sont :

- la répétition et

- la reformulation définitoire analytique lacunaire (RDAL).

La RDAL sélectionne, dans la matrice définitoire du verbe complexe, le verbe élémentaire correspondant à la description du procès. Les verbes élémentaires comme dire, regarder, attendre, aller, voir sont des verbes à très haute fréquence, peu contraignants dans leur sélection argumentale et donc plus faciles à produire que leurs équivalents distributionnels qui sélectionnent un nombre restreint d'arguments ${ }^{14}$.

Les procédures de reformulation par complexification sont :

- la paraphrase sémantique (synonymie ou ajout de précision au verbe source repris)

- la reformulation définitoire analytique complète (RDAC) qui semble se mettre en place par étapes successives (paraphrase descriptive)

- Synthétisation de deux informations exprimées par deux mots au moins, en un seul mot.

\subsubsection{Deux verbes inchoatifs tendre et soulever}

Les deux verbes suivants : tendre la boîte (4.4) et soulever le couvercle (6.2) expriment tous deux le début du procès. Dans les deux cas, l'interprétation, dans le contexte de l'histoire, correspond au procès achevé : donner la boîte et ouvrir la boîte ${ }^{15}$. Nous considérons que les verbes donner la boîte et ouvrir la boîte/le couvercle sont plus simples que tendre la boîte et soulever le couvercle du fait que les premiers réfèrent au procès dans sa globalité, ils ne sont donc pas marqués du point de vue de l'Aktionsart, et sont moins contraints du point de vue de leur sélection argumentale (on peut soulever un couvercle mais pas toutes les choses que l'on peut ouvrir). C'est cette reformulation par simplification sémantique (RDAL) que la plupart des enfants ont produit à tous les âges.

[24] Séq. 4.4. ES. (il) lui a tendu la boîte

ER. Antoine. 4 ans. (...) et puis il l'a donnée à Julie 
Quelques procédures de complexification sont cependant attestées. Un enfant de 4 ans ([25] Clara) et un enfant de 6 ans (Gaël) ont produit une reformulation complexe, relativement à leurs camarades de même âge, qui ont simplifié le verbe source ou l'ont répété. Clara reprend le verbe source tendit la boîte mais ajoute une précision directionnelle, tandis que Gaël utilise une procédure un peu plus complexe en décrivant le mouvement jusqu'à sa finalité : Tom tend le bras et Julie prend la boîte. Ces deux procédures se rapprochent de la MAD du verbe tendre (note 15) et donc d'une RDAC. À partir de 8 ans, les enfants produisent un équivalent de donner la boîte, plus complexe parce que plus spécifique (offrir) ${ }^{16}$. En effet, plus la contrainte de sélection d'un mot sur un autre est élevée, plus l'appropriation est grande, et plus il est difficile de bien associer les mots en question. Quant à Marie, elle produit le verbe converse de donner. Pour les enfants de 8 et 10 ans, la procédure est complexe parce qu'elle a lieu en deux temps, d'abord l'enfant interprète tendre la boîte en donner la boîte puis il complexifie donner la boîte en énonçant un verbe plus spécifique offrir ou converse recevoir.

[25] Séq. 4.4. ES. (il) lui a tendu la boîte

ER. Clara, 4 ans. *il lui tendit la boîte devant elle

ER. Gaël, 6 ans. Tom *tenda le bras et euh Julie *prena la boîte

ER. Charlotte, 8 ans. il l'a offert

ER. Maurine, 10 ans. il lui offrit

ER. Marie, 10 ans. quand elle l'avait reçue

La reformulation du verbe soulever [26], s'est manifestée chez quelques enfants de 8 et 10 ans par une procédure de complexification que l'on a déjà rencontrée avec le verbe guetter ([16] Daphné) : le verbe élémentaire ouvrir provenant de la RDAL du verbe source est suivi d'une précision. L'ajout d'une modalité (délicatement) réfère ici à la première phase du procès : ouvrir délicatement le couvercle: soulever le couvercle avec précaution puis continuer le mouvement jusqu'à l'ouverture.

[26] Séq. 6.2. ES. Tom souleva le couvercle

ER. Alexandre, 8 ans. il avait ouvert délicatement

ER. Paul, 10 ans. il ouvrit délicatement le couvercle

On retrouve pour ces deux verbes, marqués du point de vue de leur Aktionsart, la procédure de simplification attestée à tous les âges : la RDAL (donner, ouvrir).

Les reformulations par complexification correspondent à des tentatives de RDAC (paraphrases descriptives) à 4 et 6 ans, ensuite, à des reformulations paraphrastiques (offrir pour tendre la boîte) et à des RDAL avec indication de la modalité (ouvrir délicatement). 


\subsubsection{Quatre prédicats difficiles à restituer : grincement, tourner, éblouir, inonder}

Les quatre prédicats suivants (grincement, tourner sur lui-même, être éblouis, inonder) ont posé différents problèmes de restitution. La frontière entre les 4-6 ans d'une part et les 8-10 ans d'autre part est très nette mais surtout les procédures de complexification n'apparaissent qu'à 10 ans pour grincement, tourner sur lui-même, inonder; elles commencent chez certains enfants à 8 ans pour être éblouis.

\subsubsection{Reformulations de grincement}

Un enfant de 10 ans met en œuvre une transformation par translation que l'on peut considérer comme une procédure complexe. Le degré de complexité sémantique du prédicat nominal source et du prédicat verbal reformulé est le même. Très peu d'enfants dans chaque tranche d'âge ont répété grincement. Les enfants plus jeunes (4 et 6 ans) ont simplifié grincement en utilisant le nom générique bruit.

[27] Séq. 10.1. ES. Les enfants entendirent un grincement

ER. Romain, 10 ans. L'arbre grince

\subsubsection{Reformulations de tourner sur lui-même}

Deux procédures différentes de complexification sont ici attestées avec les enfants de 10 ans : Théo et Marion fournissent un équivalent sémantique, avec redondance chez Marion; Maxime produit une RDAC dans laquelle il analyse tourner en faire des tours (paraphrase descriptive). Les enfants plus jeunes ont simplifié syntaxiquement le verbe tourner sur luimême en supprimant le complément ou en le remplaçant par autour d'eux, autour de lui, ce qui entraîne à chaque fois un changement de sens (l'arbre tourne autour d'eux). Cette erreur de construction diminue à 8 ans (un tiers des enfants ont encore employé le verbe seul), les autres ont répété à l'identique.

[28] Séq. 10.2. ES. L'arbre était en train de tourner sur lui-même

ER. Théo, 10 ans. il pivote

ER. Marion, 10 ans. l'arbre il pivote sur lui-même

ER. Maxime, 10 ans. l'arbre fait des tours sur lui-même

\subsubsection{Reformulations de être éblouis}

Alors que les enfants les plus jeunes (4 ans) simplifient l'énoncé source en restituant uniquement la présence de la lumière, et que quelques enfants de 6 ans parviennent à répéter 
le verbe au passif, les procédures de complexification sont attestées à partir de 8 ans. Un enfant de 8 ans (Charlotte) produit un prédicat nominal, plus ou moins équivalent dans le contexte, à la nominalisation du verbe : éblouir > éblouissement > éclairement. Deux enfants de 10 ans produisent une translation (Marc et Océanie): furent éblouis > éblouissante (transformation) avec un changement de sens chez Océanie qui « récupère » la métaphore exprimée par le verbe inonder de la relative (lumière qui inondait l'intérieur de l'arbre) tout en la déconstruisant, l'élément liquide est à interpréter dans son sens concret dans la reformulation d'Océanie. Cette dernière reformulation est particulièrement complexe même si le sens est modifié. Alexandrine transforme le verbe source à la voix active.

Les transformations ne sont pratiquement jamais attestées avant 8 ans, ce qui nous invite à conclure que ce type de reformulation est complexe.

[29] Séq. 11.1. ES. Les enfants furent éblouis par la lumière

ER. Camille, 4 ans. ils ont vu de la lumière

ER. Pauline, 4 ans. il y avait de la lumière

ER. Charlotte, 8 ans. il y a eu un espèce de ... un éclairement

ER. Marc, 10 ans. il y a eu une lumière éblouissante

ER. Océanie, 10 ans. et ils voient une mer éblouissante

ER. Alexandrine, 10 ans. une grande lumière l'éblouit

\subsubsection{Reformulations de inonder}

La relative est restructurée par Léo. Cette transformation par restructuration est encore plus complexe à réaliser que les transformations que nous avons vues en [29] (translations et transformation active) dans la mesure où les restructurations sont contraintes par le lexique et n'ont donc pas le caractère (relativement) systématique des transformations ${ }^{17}$. Aucun enfant de 4, 6 et 8 ans n'a reformulé d'une façon ou d'une autre la métaphore verbale.

[30] Séq. 11.2. ES (lumière) qui inondait l'intérieur de l'arbre

ER. Léo, 10 ans. c'est inondé de lumière

\subsubsection{Reformulations du verbe opérateur sembler}

Les reformulations de ce verbe, qui s'applique à la suite se parler en chantant, sont représentatives de la complexification des reformulations. Aucun enfant de 4 ans ne l'a reformulé (alors que 12 enfants de cette tranche d'âge sur 15 ont reformulé la séquence 12) :

[31] Séq. 12. ES. Les fleurs semblaient se parler en chantant 
ER. Alexia, 6 ans. on dirait que les fleurs elles chantaient

ER. Chloé, 6 ans. Ils ont l'impression que les fleurs elles se parlent en chantant

ER. Solène, 8 ans. On disait même qu'elles parlaient en chantant

ER. Alexandre, 8 ans. On sentait qu'il y avait des fleurs qui discutaient en chantant

ER. Antonin, 8 ans. On croyait que les fleurs se parlaient en chantant

ER. Charlotte, 8 ans. C'est comme si les fleurs ils parlaient ensemble

ER. Claire, 8 ans. avec des fleurs qui paraissaient chanter

ER. Marc, 10 ans. les fleurs qui avaient l'air de se parler

ER. Barbara, 10 ans. On avait l'impression que les fleurs se parlaient en chantant

ER. Marion, 10 ans. Il lui semble que les fleurs se parlent entre elles

Les deux enfants de 6 ans qui ont reformulé sembler ont produit chacun un équivalent sémantique de même degré de complexité (sémantique), et l'ont construit avec une complétive. À 8 ans, les enfants ont été nombreux à tenter de reformuler ce verbe mais seules Charlotte et Claire ont fourni un équivalent sémantique (même degré de complexité) construit autrement qu'avec une complétive et autrement que dans le texte source également. À 10 ans, les enfants sont un peu moins nombreux que les 8 ans à avoir reformulé le verbe sembler mais tous l'ont reformulé de façon équivalente. Marion reprend le même verbe et le transforme à la forme impersonnelle.

L'évolution quantitative distingue les 6 ans des 8-10 ans. La diversité des tentatives des 8 ans montre bien que les enfants cherchent à s'approprier la langue par des procédures de reformulation, toujours nombreuses à 8 ans et, dans certains cas déjà nombreuses à 6 ans, mais la production d'un équivalent sémantique, d'une transformation ou d'une restructuration n'est attestée qu'à 10 ans.

L'analyse des reformulations des quatre prédicats ci-dessus ainsi que du verbe opérateur sembler confirment les résultats précédents et montrent de nouvelles procédures de complexification et de simplification. Les reformulations par simplification correspondent à :

- La suppression d'un des deux prédicats (seule l'existence de la lumière est mentionnée mais plus comme agent du verbe passif furent éblouis, qui est supprimé)

- La suppression du complément verbal (dans tourner sur lui-même) ce qui entraîne un changement de sens.

- La répétition (grincement, tourner sur lui-même, être éblouis)

- L'emploi d'un terme générique (grincement > bruit)

Les reformulations par complexification correspondent à : 
- Des paraphrases sémantiques (tourner sur lui-même, sembler)

- Des paraphrases descriptives (RDAC) : tourner sur lui-même > faire des tours sur luimême

- des transformations par translation (grincement, être éblouis), à la forme impersonnelle (semblaient > il lui semble), à la forme active (être éblouis)

- des transformations par restructuration et reprise de la métaphore (inonder).

En conclusion, si l'absence de reformulation et la répétition sont des procédures plus faciles à réaliser que la production d'une équivalence sémantique, celle-ci prend des formes qui mettent en œuvre des procédures de plus en plus complexes en fonction de l'âge. La reformulation des prédications qui contiennent un mot supposé complexe a produit au niveau de ce mot des RDAL à tous les âges. Celles-ci diminuent systématiquement à 10 ans pour faire place à des reformulations analytiques définitoires complètes (paraphrases descriptives), mais aussi à des procédures de synthétisation ou de condensation (deux mots reformulés par un seul), il y a aussi une reformulation de la métaphore. Au niveau de la phrase, la présence de ces mots complexes a produit, outre des paraphrases descriptives, des paraphrases formelles : transformations et restructurations. Dans chaque cas, assez peu d'enfants de 10 ans ont produit ces reformulations complexes mais ce qui est déterminant, c'est qu'aucune de ces procédures ne soit attestée avant 10 ans. À partir de 6 ans apparaissent pour certains mots des synonymes mais ce n'est qu'à 8 ans que la paraphrase sémantique se généralise, les 6 ans préférant majoritairement la répétition tandis que les 8 ans expérimentent diverses procédures.

\subsection{Complexité syntaxique}

Analysons maintenant un échantillon de prédications qui ont présenté quelques difficultés soit aux enfants de 4 ans seulement soit à tous, lors de la restitution de l'histoire. Pour mesurer la difficulté dans la reformulation de ces prédications, nous avons utilisé le nombre de reformulations paraphrastiques que l'ensemble des enfants a produit (Martinot, sous presse) : plus le nombre de reformulations paraphrastiques est réduit, plus la prédication est supposée difficile à restituer sous une forme ou une autre ${ }^{18}$. L'objectif de cette analyse est de repérer quels types de reformulations paraphrastiques sont produits par les enfants des 4 tranches d'âges pour des énoncés sources présentant des degrés de complexité différents.

Séq. 1.1. elle tenait par la main une petite fille (30 reformulations paraphrastiques, soit $50 \%$ de tous les enfants)

Séq. 1.2. (...) que personne n'avait encore jamais vue (15 ref. paraph., soit $25 \%$ de tous les enfants) 
Séq. 3. (3.1) Tom était fou de joie (3.2) à l'idée d'avoir peut-être une nouvelle amie (15 ref. paraph. soit $25 \%$ de tous les enfants)

Séq. 13. (je veux apprendre à parler avec les oiseaux) qui savent tout ce qui se passe dans le ciel (12 ref. paraph., soit $20 \%$ de tous les enfants)

Séq.6.2 je t'attends ce soir à 8 h sous le gros arbre à l'entrée de la forêt (8 ref. paraph. Soit $13 \%$ de tous les enfants)

\subsubsection{Cas « contrôle » d'une prédication syntaxiquement simple}

La phrase suivante [32] n'est pas complexe et constitue dans notre analyse une sorte de phrase contrôle. Elle fait partie de celles qui ont été le plus paraphrasées ${ }^{19}$. Cependant, elle a réservé des difficultés aux enfants de 4 ans. 8 sur 15 ont en effet changé le sens de cette phrase en utilisant une autre préposition :

[32] Séq. 1.1. ES. elle tenait par la main une petite fille

ER. Laurana, 4 ans. la maîtresse tenait dans sa main une petite fille

L'écart le plus important dans les reformulations paraphrastiques de la séquence (1.1) se situe entre 4 et 6 ans (les 6 ans ne sont plus que quatre à avoir employé une préposition inappropriée).

Cette séquence a été paraphrasée essentiellement de deux façons : soit par une réduction de la prédication à un complément prépositionnel en avec une petite fille :

[33] Séq. 1.1. ES. (la maîtresse est arrivée dans la cour de l'école plus tard que d'habitude.) Elle tenait par la main une petite fille

ER. Antoine, 4 ans. la maîtresse arriva en retard avec une petite fille, procédure qui diminue progressivement entre 4 et 8 ans et disparaît à 10 ans. Soit par une restructuration du verbe, dont le nombre est stable de 6 à 10 ans :

[34] ER. Camille, 4 ans. une maîtresse tenait la main d'une petite fille

ER. Jean-Alexandre, 6 ans. la maîtresse tenait une petite fille à la main

ER. Paul, 10 ans. elle tenait à la main une petite fille

On peut donc considérer que, lorsque la phrase source est simple (cas de la séq. 1.1), la réduction de cette phrase à un complément prépositionnel, qui devient une prédication seconde de la phrase précédente (cf. Antoine [33]) - ce qui complexifie la phrase d'accueil de même que les différentes restructurations du verbe (cf. [34]), sont des procédures qui semblent faciles à appliquer.

De telles procédures de reformulation, a priori complexes à mettre en ouvre (réduction et restructuration), nous informent donc de la complexité relative des phrases dans la langue, 
telle qu'elle est «perçue » par les locuteurs : si dès 4 ans, un enfant peut reformuler un énoncé de type (1.1) par une restructuration, alors cet énoncé peut être considéré comme simple ${ }^{20}$. Cela peut également signifier que les reformulations de l'ES sont plus familières (quel que soit leur degré de complexité syntaxique) que l'ES. Mais cela signifie dans tous les cas que les énoncés reformulés font bel et bien partie du stock d'énoncés que maîtrise l'enfant.

Cependant, à partir de 6 ans, sont attestés, pour la phrase (1.1), d'autres modes de reformulation : (a) paraphrases sémantiques (1 à 6 ans, 2 à 8 ans, 4 à 10 ans), (b) permutation et pronominalisation (2 à 8 ans), restructuration du verbe à la forme gérondive (1 à 10 ans), qui n'étaient attestés qu'à partir de 8 ans dans les exemples contenant un mot lexical complexe (cf. partie précédente consacrée à la complexité lexicale) :

[35] Séq. 1.1. ES. elle tenait par la main une petite fille

ER. Rémy, 6 ans. elle ramenait une petite fille avec elle (a)

ER. Ninon, 8 ans. elle tenait par la main un enfant (a)

ER. Romain, 10 ans. elle tient par le bras une petite fille (a)

ER. Solène, 8 ans. (...) qui tenait une petite fille par la main (permutation) (b)

ER. Léa, 8 ans. la maîtresse elle la tient par la main (pronominalisation) (b)

ER. Barbara, 10 ans. (...) en tenant une petite fille par la main (gérondif + permutation) (b)

\subsubsection{Reformulations de deux relatives explicatives}

Séq. 1.2. (...) que personne n'avait encore jamais vue

Séq. 13. (je veux apprendre (...) oiseaux) qui savent tout ce qui se passe dans le $\operatorname{ciel}^{21}$

Les prédications (1.2) et (13) sont des prédications secondes (relatives explicatives) respectivement situées en début et en fin d'histoire. Aucun enfant de 4 ans ne produit de paraphrase de la séquence (13) et un seul en produit pour la séquence (1.2). La prédication (13) (20\% de paraphrases) est (probablement) plus complexe que la prédication (1.2) (25\% de paraphrases) du fait que la relative qui savent tout ce qui se passe dans le ciel contient une complétive : tout ce qui se passe dans le ciel $^{22}$. Mais ce n'est pas ce phénomène qui semble avoir réservé des difficultés aux enfants mais bien plutôt le fait que le sujet de savent n'est pas le même que le sujet du verbe précédent : je (Tom) veux apprendre à parler, puisque presque la moitié des enfants de 6 et de 8 ans ont modifié le sens de l'ES en disant :

[36] ER. Madeleine, 6 ans. je veux savoir parler avec les oiseaux pour savoir tout ce qui se passe dans le ciel 
Les paraphrases de la prédication :

[37] Séq. 1.2. (...) que personne n'avait encore jamais vue

se répartissent en trois types de paraphrases qui correspondent au groupe des 4-6 ans, au groupe des 8 ans et au groupe des 10 ans. Quantitativement, les 8 et les 10 ans fournissent trois fois plus de paraphrases que les plus jeunes.

Les 4 et 6 ans synthétisent la relative que personne n'avait encore jamais vue en un adjectif sémantiquement équivalent :

[38] ER. Sacha, 6 ans. ce matin la maîtresse tenait la main à une nouvelle élève

À 8 ans, la synthétisation de la relative se poursuit (3 enfants) mais un nouvel adjectif apparaît :

[39] ER. Océane, 8 ans. il y a une petite fille inconnue

Trois autres enfants reformulent la relative par une paraphrase sémantique du fait de l'introduction d'un verbe synonyme :

[40] ER. Rémy, 8 ans. (...) qu'on ne connaissait pas

ou d'un nom qui lexicalise personne :

[41] ER. Ninon, 8 ans. (...) que les élèves n'avaient encore jamais vu

À 10 ans, plusieurs enfants modifient le point de vue (Julie devient le sujet grammatical en même temps que l'agent) tout en produisant une paraphrase fonctionnelle (procédure majoritaire à 10 ans) :

[42] ER. Maurine, 10 ans. c'est l'histoire d'une petite fille qui vient pour la première fois dans une école

ER. Théo, 10 ans. c'est Julie qui entre dans une nouvelle école

ER. Marie, 10 ans. un jour une nouvelle fille arrive dans une école

ER. Maxime, 10 ans. il était une fois une petite fille qui faisait sa rentrée à l'école

La paraphrase sémantique à partir de 8 ans et la paraphrase fonctionnelle à partir de 10 ans peuvent être considérées comme des procédures de complexification. D'une part, l'introduction d'un lexique équivalent signale que l'enfant dispose de plusieurs énoncés pour donner la même information, d'autre part la paraphrase fonctionnelle, équivalente en contexte seulement, signale ici que l'enfant s'approprie le sens en modifiant le point de vue du texte source.

Les paraphrases de la prédication : 
[43] Séq. 13. (je veux apprendre (...) oiseaux) qui savent tout ce qui se passe dans le ciel

se répartissent en deux groupes (les 4 ans n'ont pas fourni une seule paraphrase). À 6 et 8 ans, les paraphrases sont pratiquement les mêmes : restructurations par effacement de la complétive (ce qui se passe), paraphrases sémantiques par substitution de il y a à se passe, reprise du relatif dans quatre cas, substitution de parce que dans un cas :

[44] ER. Margot, 6 ans. avec les fourmis qui savent tout sur la terre

ER.Antonin, 8 ans. aux oiseaux qui savent tout dans le ciel

ER.Gaël, 6 ans \& Claire, 8 ans. aux oiseaux qui savent tout ce qu'il y a dans le ciel

ER.Océane, 8 ans. aux oiseaux parce qu'ils savent tout dans l'air

mais elles sont peu nombreuses : 2 à 6 ans, 3 à 8 ans.

À 10 ans, non seulement le nombre de reformulations paraphrastiques augmente nettement, mais elles introduisent un nouveau lexique :

[45] ER. Théo, 10 ans. parler aux oiseaux parce qu'ils connaissent tout de l'air Le nombre des restructurations par effacement de la complétive avec reprise du relatif augmente nettement à 10 ans, ainsi que l'emploi des connecteurs de cause (parce que, car). Enfin, un enfant produit une reformulation très complexe qui restructure la prédication principale parler avec les poissons et la prédication seconde qui savent tout ce qui se passe dans l'eau:

[46] ER. Maxime, 10 ans. je voudrais pouvoir parler aux poissons de tout ce qu'ils savent dans l'eau.

Le verbe parler est restructuré (parler avec les poissons > parler aux poissons de qch) puisqu'il acquiert un nouveau complément : le verbe savoir. Les poissons savent tout ce qui se passe devient parler de tout ce qu'ils savent. Dans l'énoncé source, tout (ce qui se passe) est le complément de savoir, dans l'énoncé reformulé, le complément de savoir est aussi le complément de parler.

La relative de la séquence (13) fait partie des énoncés très peu paraphrasés mais également peu répétés : 1 enfant à 4,6 et 8 ans. À 10 ans, en revanche, 3 enfants ont strictement répété la relative alors que la procédure de répétition n'est pratiquement plus attestée dans cette tranche d'âge. Nous verrons ci-dessous la raison de ces répétitions. La séquence (13) a pourtant été reformulée par la quasi-totalité de tous les enfants quel que soit leur âge. La difficulté à produire une reformulation paraphrastique s'explique par la complexité syntaxique de 
l'ensemble de la séquence (13) et plus particulièrement par la difficulté à distinguer 2 actants : celui qui veut apprendre à parler et ceux qui savent tout ....

Une autre difficulté tient à la très faible plasticité paraphrastique de toute la séquence (13) : l'essentiel des modifications a porté sur les deux premiers verbes (Je veux apprendre à > je veux savoir, je voudrais pouvoir), sur la substitution de il y a à se passe et sur la complétive qui a été effacée. C'est la faible plasticité paraphrastique qui explique que 3 enfants de 10 ans aient répété la relative. Devant une phrase complexe et peu facilement paraphrasable, les 5 enfants de 4 ans ont changé le sens, les autres n'ont pas reformulé du tout la relative, à 6 et 8 ans, la majorité a modifié le sens de l'énoncé source, et seulement 2 enfants de 6 ans et 3 de 8 ans ont produit un équivalent sémantique. L'augmentation spectaculaire des paraphrases à 10 ans, la forte diminution des changements de sens ( 2 enfants de 10 ans seulement) et le fait que 3 enfants aient réussi à répéter cette prédication complexe signifie que l'augmentation du nombre des paraphrases est sans doute le critère le plus pertinent pour évaluer le degré d'acquisition de la langue maternelle, même si, dans ce cas précis, les procédures de reformulation paraphrastique sont les mêmes à 6,8 et 10 ans, à l'exception d'un enfant de 10 ans qui a restructuré les 2 prédications (principale et relative). Il faut ajouter que l'augmentation très forte des paraphrases entre 8 et 10 ans est corrélée, dans la séquence (13), à l'augmentation du nombre des relatives et subordonnées introduites par un connecteur introduisant une relation causale (ces dernières sont fonctionnellement équivalentes aux relatives dans cette séquence).

\subsubsection{Reformulations d'une prédication complexe à 3 opérateurs successifs}

[47] Séq. 3. (3.1) Tom était fou de joie (3.2) à l'idée d'avoir peut-être une nouvelle amie

Cette prédication complexe a été reformulée par un quart des enfants, et dans les mêmes proportions pour les quatre tranches d'âge que la prédication (1.2) (que personne n'avait encore jamais vиe) que nous avons commentée ci-dessus. La complexité syntaxique de ces deux prédications ne provient cependant pas des mêmes phénomènes. Si l'ensemble des enfants a reformulé le sentiment de très grande joie (3.1) à partir de 6 ans (un enfant de 4 ans a fait de même) :

[48] ER. Camille, 4 ans. il est très content

ER. Margot, 6 ans. Tom était très heureux

ER. Léa, 8 ans. Tom était ravi 
assez peu d'enfants ont paraphrasé l'ensemble de la séquence (3) (2 enfants de 6 ans, 7 de 8 ans et 6 de 10 ans). Parmi ces enfants, la construction de l'adjectif opérateur (fou de joie à...) paraphrasée le plus souvent par très/tout content de, augmente nettement entre 6 et 8 ans. Un seul enfant de 6 ans a paraphrasé l'ensemble de la séquence source avec l'adjectif opérateur (Achille) contre 4 à 8 ans :

[49] ER. Achille, 6 ans. Tom était très excité d'avoir une nouvelle amie

Anne, 6 ans. Super je vais me faire une nouvelle copine

Ilan, 8 ans. Tom était très content d'avoir une petite copine

La seconde difficulté de la séquence (3) se trouve dans la présence d'un second opérateur à l'idée de. Ce dernier nom opérateur est reformulé par un seul enfant de 8 ans :

[50] ER. Marc, 8 ans. Tom était ravi parce qu'il croyait qu'il allait se faire ami.

La construction complexe (à l'idée d'avoir...) est reformulée plus souvent à 10 ans (3 enfants) :

[51] ER. Marc, 10 ans. Tom était tout content à l'idée d'avoir une nouvelle copine

ER. Marion, 10 ans. Tom il est tout content à l'idée qu'il pourra se faire peut-être une nouvelle camarade de classe

ER. Léo, 10 ans. Tom était très content il pensait avoir peut être une nouvelle amie À 8 et 10 ans, 3 enfants de chaque tranche d'âge expriment l'éventualité de la nouvelle relation amicale entre les enfants :

[52] ER. Anthony, 8 ans. (...) content de pouvoir avoir une nouvelle amie

ER. Maurine, 10 ans. Tom est très content d'avoir une nouvelle voisine et peut-être sa nouvelle copine

Les difficultés de paraphrasage de la séquence (3) tiennent davantage à la succession de deux constructions complexes (adjectif opérateur s'appliquant à un nom opérateur) qu'à l'interprétation de cette séquence. On peut classer ces difficultés selon un ordre croissant :

1/Reformulation de l'ensemble, à partir de 6 ans

2/Reformulation paraphrastique avec adjectif opérateur, à partir de 6 ans ( 1 seul cas de paraphrase, 2 cas avec changement de sens)

3/Reformulation paraphrastique de l'éventualité, avec verbe opérateur ou reprise du même adverbe à partir de 8 ans

4/Reformulation paraphrastique avec nom opérateur, à partir de 10 ans

Dans cette séquence, la paraphrase sémantique pour chacune des deux prédications (3.1) et (3.2) est largement majoritaire (une paraphrase fonctionnelle à 6 , à 8 et à 10 ans, cf. [49] Anne, 6 ans). La différenciation dans le degré de complexité ne nous est pas révélée ici par le type de paraphrase utilisé par les enfants mais par le statut syntaxique des composants de la 
séquence : la reformulation des deux prédications ne commence qu'à 6 ans, celle de l'adjectif opérateur n'a qu'une attestation à 6 ans, celle du nom opérateur apparaît comme l'opération la plus complexe (10 ans seulement).

\subsubsection{Reformulation d'une prédication comprenant une accumulation de compléments}

[53] Séq.6.2 je t'attends ce soir à 8h sous le gros arbre à l'entrée de la forêt

Cette prédication a réservé des difficultés redoutables lors de sa reformulation. 13\% seulement de tous les enfants l'ont paraphrasée. Elle est pourtant construite à partir d'un verbe ne présentant aucune difficulté ni syntaxique ni sémantique mais l'ensemble des informations apporté par les compléments est complexe. Deux compléments réfèrent au temps ponctuel, les deux suivants à la localisation. Le second complément temporel précise le premier, non nécessaire à l'information étant donné la situation d'énonciation qui implique que c'est le soir même que les protagonistes de l'histoire doivent se retrouver. Les deux compléments locatifs sont en revanche nécessaires, mais leur ordre d'énonciation est l'inverse du repérage spatial effectif qu'il faut faire : il faut d'abord aller à l'entrée de la forêt et ensuite repérer un certain arbre.

Tous les enfants ont exprimé au moins une information temporelle et une information locative mais très peu ont réussi à reformuler l'information complète équivalente à : 8h (sous + auprès de + à) le (gros + grand) (arbre + chêne) (à l'entrée de + au début de + devant) la forêt.

Dès 4 ans, 3 enfants sur 5 ont restitué l'heure précise, tous les enfants plus âgés l'ont fait. La restitution de l'information locative complète a posé de nombreux problèmes du fait qu'il faut à la fois décrire l'arbre (deux sur cinq l'ont fait à 4 ans, 7 sur 12 à 6 ans, 6 sur 12 à 8 ans, 10 sur 13 à 10 ans) et le situer précisément par rapport à la forêt.

A l'entrée de la forêt a été reformulé par 3 enfants sur 5 de 4 ans par près du premier arbre (E + de la forêt $)^{23}$, les autres ont simplement dit dans la forêt.

À 6,8 et 10 ans, 3 enfants seulement de 6 et 8 ans, 4 de 10 ans ont répété à l'entrée de la forêt, ou ont paraphrasé par (au début + à la porte + devant) la forêt .

Pour résumer, à 4 ans, aucun enfant ne parvient à donner l'information complète, soit parce que l'heure précise manque, soit parce que la construction à l'entrée de la forêt ne fait pas sens (les énoncés les plus approchants étant : près du premier arbre de la forêt). De 6 à 10 ans, la mention temporelle indispensable est indiquée par tous les enfants mais très peu fournissent l'information locative première (l'entrée de la forêt): 
[54] ER. Margot, 6 ans.Tom je t'attends à $8 \mathrm{~h}$ du soir près du grand arbre près de la forêt (changement de sens)

ER. Ilan, 8 ans. rendez-vous ce soir à $8 \mathrm{~h}$ à l'arbre à la porte de la forêt (manque la description de l'arbre)

ER. Léo, 10 ans. ça disait à $8 \mathrm{~h}$ au grand chêne devant la forêt (paraphrase sémantique approximative)

Les procédures de reformulation des cinq prédications analysées ci-dessus correspondent à des procédures de simplification quand il y a effacement (prédications secondes, complétive et complément locatif ou temporel nécessaire pragmatiquement), transformation d'une relative en adjectif, réduction du nombre des actants, remplacement d'un mot moins fréquent par un mot plus fréquent (fou de joie > très content, se passe > il y a) et enfin répétition.

Ces procédures de simplification sont attestées à tous les âges, mais elles sont moins fréquentes à 8 et 10 ans et ne sont attestées que lorsqu'elles n'entraînent pas de changement de sens.

Les procédures de reformulation des cinq prédications correspondent à des procédures de complexification, attestées plus ou moins tôt en fonction de la plasticité paraphrastique de la prédication source : quand cette plasticité est grande, alors des restructurations et quelques paraphrases sémantiques et fonctionnelles sont attestées chez les 4-6 ans, quand la plasticité paraphrastique est réduite alors seuls les 10 ans ont produit des transformations et restructurations complexes (transformation au gérondif, restructuration de deux prédications), des paraphrases fonctionnelles avec changement de point de vue, des paraphrases sémantiques reprenant le relatif ou produisant un équivalent fonctionnel, et en général des paraphrases reprenant toutes les informations des énoncés sources (exprimées par différentes modalités, par plusieurs opérateurs successifs, par différents compléments locatifs et temporels).

\section{Conclusion}

Malgré les diverses hypothèses avancées sur le processus d'acquisition de la langue maternelle, on doit bien admettre qu'une explication consensuelle fait encore défaut dans de nombreux domaines de l'acquisition linguistique.

L'étude qui est rapportée ici avait un double objectif : d'une part, apporter de nouveaux arguments à la théorie acquisitionnelle de la reformulation selon laquelle l'enfant acquiert la langue, ensemble plus ou moins vaste constitué d'énoncés, en transformant, selon un principe stable de reprise associée à un changement, les énoncés de la langue cible qu'il a mémorisés, et d'autre part, classer les procédures de reformulation paraphrastique dans un ordre qui 
suppose que certaines paraphrases sont plus difficiles à mettre en œuvre que d'autres et que c'est cette mise en œuvre qui accroît le degré de complexité d'un ensemble d'énoncés et progressivement de la langue. La difficulté relative de cette mise en œuvre est en partie déterminée par la complexité relative de l'énoncé source, quel que soit le critère de complexité retenu, mais aussi par la nature même de la paraphrase appliquée à l'énoncé source. Ainsi, on peut classer par ordre de difficulté croissant les reformulations paraphrastiques attestées dans l'expérimentation :

1. Paraphrases descriptives correspondant d'abord à des reformulations définitoires analytiques lacunaires (RDAL, dans ce cas, la reformulation simplifie l'énoncé source, et la relation paraphrastique n'est pas garantie: guetter \# attendre) puis à des reformulations définitoires analytiques complètes (RDAC).

2. Paraphrases sémantiques

3. Paraphrases formelles : transformations puis restructurations

4. Paraphrases fonctionnelles

Il reste à expliquer pourquoi certaines paraphrases sont plus complexes à produire que d'autres et à confirmer, sur un plus grand nombre d'énoncés présentant d'autres facteurs de complexité, ces résultats.

Etant donné la tâche expérimentale demandée aux enfants, on aurait pu s'attendre à ce que l'essentiel des reformulations produites simplifie les énoncés sources correspondant. Nos résultats montrent la tendance inverse. Certes, les enfants de 4 ans simplifient souvent les énoncés sources en effaçant un mot ou une construction complexe (la relative par exemple), en répétant plutôt qu'en paraphrasant les prédications simples, en réduisant le nombre des actants, en produisant des mots plus courants ou moins contraints mais d'une part quelques tentatives de complexification (par rapport aux simplifications précédentes) sont déjà attestées: RDAC et restructurations d'une prédication simple. D'autre part, dès 6 ans, quelques reformulations maintiennent le même degré de complexité que dans l'énoncé source (cas des paraphrases sémantiques et des RDAC) et à partir de 8 ans, les procédures de reformulation paraphrastique qui maintiennent ou même augmentent le degré de complexité de l'énoncé source (synthétisation de deux informations en un mot, par exemple) sont significativement plus nombreuses. Enfin, certains types de restructuration (portant sur deux prédications en même temps) de même que la reprise de la métaphore (à la fin de l'histoire), ou la complétude d'informations temporelles et locatives, correspondant à de hauts degrés de complexité syntaxique et sémantique, sont attestés à 10 ans. 
Le protocole expérimental que nous utilisons (lecture puis restitution immédiate d'une histoire) et l'objectif que nous poursuivons (la mise à jour des procédures de reformulation entre des enfants d'âges différents, voire de langues différentes) fournissent un outil d'observation original dans lequel l'input est contrôlé. La comparaison systématique, transversale et longitudinale, permet, quant à elle, d'obtenir des résultats inédits sur le mode de complexification de la langue en acquisition au cours de la période des acquisitions dites tardives. Le taux et le type de paraphrases produites pour un énoncé donné fournissent en effet un nouvel outil de mesure de la complexité de cet énoncé, telle qu'elle est perçue, et en tous cas, produite par le locuteur.

À ce jour, aucune étude sur le ou les processus de complexification n'a été menée chez les enfants. Ce type d'étude permettrait peut être aussi de répondre à la question de pourquoi les langues se complexifient en diachronie (Barker \& Pederson, 2009 : 392).

\section{Références}

BARKer M. et PEDERSON E., 2009, «Syntactic complexity vs. concatenation in a verbal production task», in GIVÓN T. et ShIBATANI M., Syntactic complexity, Amsterdam/Philadelphia, John Benjamins, p. 391-403.

BlAnCHE -BENVENISTE Cl. et al., 1990, Le français parlé : études grammaticales, Paris, Editions du CNRS.

CAdiot P. et Furukawa N., 2000 (éds.), La prédication seconde, Langue française, 127.

Chomsky N., 1995, Minimalism, Cambridge, MA., MIT Press.

CHOMSKy N., 2000, New horizons in the study of language and mind, Cambridge, MA, MIT Press.

FuruKaWA N., 1996, Grammaire de la prédication seconde, Louvain-la-neuve, Duculot.

Gerolimich S., Martinot Cl., De Gioia M., 2009, «Reformulations définitoires spontanées chez des francophones et des italophones de 6, 8 et 10 ans », Autour de la définition, 6, http:// publifarum.farum/it/ezine_printarticle.php?art_id=149.

Givón T., 1995, Functionalism and grammar, Amsterdam \& Philadelphia, John Benjamins. Givón T., 2009, « introduction », in Givón T et ShiBATANi M., Syntactic complexity, Amsterdam/Philadelphia, John Benjamins, p. 1-19. 
GLEITMAN L.R, WANNER E., 1982, «Language acquisition : the state of the art », in WANNER E. et GleitMan L.R., language and acquisition : the state of the art, Cambridge, Cambridge University Press.

GOLDBERG A., 1995, Constructions. a construction grammar approach to argument structure, Chicago, University of Chicago Press.

GRoss, G., 2010, «Les verbes supports et l'actualisation des prédicats nominaux », in IBRAHIM A.H., Supports et prédicats non verbaux dans les langues du monde, Paris, CRL p. $16-35$

Gross M., 1975, Méthodes en syntaxe, Paris, Hermann.

Gross M., 1981, «Les bases empiriques de la notion de prédicat sémantique », Langages, 63, p. 7-52.

Gross M., 1994, «La structure d'argument des phrases élémentaires », Lingua franca, 1, Schena editore.

HARRIS Z. S., 1969, “The two systems of grammar: report and paraphrase", Transformation and discourse analysis papers 79 [p. 612-92 in papers].

HARRIS Z. S., 1976, Notes du cours de syntaxe, Paris, Seuil.

HARRIS Z.S., 1988, 2007 (trad.fr), Language and information, New York, Columbia University Press (traduction française : IBRAHIM A.H.. et MARTINOT Cl., La langue et l'information, Paris, CRL.)

IBRAHIM A.H., (éd.) 1996, Les supports, Langages, 121.

IBRAHIM A.H., 2001, «Argumentation interne et enchaînements dans les matrices définitoires », Langages, 142, p.92-126.

IBRAHIM A.H., 2007, « introduction », la langue et l'information, paris, crl, p. 3-26.

IBRAHIM A.H., 2009, « les conditions de la prédication dans les langues », in IBRAHIM A.H., Prédicats, prédication et structures prédicatives, Paris, CRL, p. 12-49.

IBRAHIM A.H., 2010, « Supports d'actualisation et dualité constitutive de la prédication », in IBRAHIM A.H., Supports et prédicats non verbaux dans les langues du monde, Paris, CRL, p. 6-73.

IBRAHIM A.H et MARTINOT Cl., 2004, «Les reformulations matricielles lacunaires des enfants », Travaux de linguistique, 48, p. 21-40.

LE Ny, J.-F., 2005, Comment l'esprit produit du sens, Paris, Odile Jacob. 
LiEven E. et Tomasello M., 2008, « Children's first language acquisition from a usagebased perspective », in ROBINSON P. et \& Ellis N., Handbook of cognitive linguistics and second language, New York et Londres, Routledge, Acquisition, p. 168-196.

MARTINOT Cl., 1994, La reformulation dans des productions orales de définitions et explications. (enfants de maternelle), Thèse de doctorat, dir. Blanche-Noëlle Grunig, Université Paris VIII..

MARTinOt Cl., 1996, « Prédicats et supports chez un enfant de 3 ans », Langages, 121, p.73-90.

MARTINOT Cl., 1997, «Les verbes supports dans l'acquisition de la syntaxe », in MARTINOT Cl., Actes du colloque international sur l'acquisition de la syntaxe en langue maternelle et en langue etrangère, Annales littéraires de l'université de Ffranche-Comté, 631, p. 87-105.

MARTINOT Cl., 2000, «Etude comparative des processus de reformulation chez des enfants de 5 à 11 ans », Langages, 140, p. 92-123.

MARTINOT Cl., 2003, «Les acquisitions tardives en français langue maternelle », in

MARTINOT Cl., et IBRAHIM A.H., La reformulation : un principe universel d'acquisition, Paris, Kimé, p. 39-69.

MARTinot Cl.,, 2005, Comment parlent les enfants de 6 ans ? pour une linguistique de l'acquisition, Besançon, Presses universitaires de Franche-Comté.

MARTINOT Cl.,, 2007, « quand acquisition rime avec reformulation : nécessité d'une réponse linguistique aux phénomènes d'acquisition de la langue maternelle », recherches linguistiques, 29, p. 179-211.

MARTINOT Cl., sous presse, «Reformulations paraphrastiques et stades d'acquisition en français langue maternelle », Cahiers de Praxématique, 52.

Martinot Cl., Gerolimich S., PAProcka-Piotrowska U. et Sowa M., 2008,

« Reformuler pour acquérir sa langue maternelle ? investigation auprès d'enfants français, italiens et polonais de 6, 8 et 10 ans », in SCHUWER M., LE BOT M.-C., RICHARD E., Pragmatique de la reformulation, Presses universitaires de Rennes, p. 221-240.

Martinot Cl., Kuvac-Kraljevic J., Bosnjak-Botica T. et Chur L., 2009, «Prédication principale vs seconde à l'épreuve des faits d'acquisition », in IBRAHIM A.H., Prédicats, prédication et structures prédicatives, Paris, CRL, p. 50-81. 
MARTINOT Cl., Gerolimich S., (sous presse) «Acquisition de la compétence paraphrastique par des enfants francophones et italophones de 4 à 10 ans », La synonymie audelà du lexique, éd. Forum, Université de Udine.

TCHEKHOFF C., 1977, «La prédication », Langue française, 35, p. 47-57.

Tomasello M., 2003, Constructing a language, Cambridge, MA, Harvard University Press.

Annexe

\section{Texte source : Tom et Julie}

1) Ce matin-là, la maîtresse est arrivée dans la cour de l'école plus tard que d'habitude. Elle tenait par la main une petite fille que personne n'avait encore jamais vue.

2) Arrivée en classe, la maitresse a dit: «Les enfants, je vous présente votre nouvelle camarade, elle s'appelle Julie. Tom, la place est libre à côté de toi, Julie sera ta voisine, sois bien gentil avec elle !»

3) Tom était fou de joie à l'idée d'avoir peut être une nouvelle amie. Le soir, chez lui, il a fabriqué une petite boîte ronde, rouge et dorée, pour Julie.

4) Le lendemain matin, dans la cour de l'école, Tom guettait l'arrivée de sa nouvelle petite voisine. Dès qu'il l'a aperçue, il s'est dirigé vers la fillette et lui a tendu la boîte qu'il avait fabriquée pour elle, la veille.

5) Julie aimait tellement cette boîte qu'elle la prenait toujours avec elle. Quand la maitresse disait : « Sortez vos affaires ! », Julie posait délicatement la boîte entre Tom et elle, sur leur table de travail.

6) Un jour, Julie chuchota à Tom : «Ouvre la boîte ! » Tom souleva le couvercle et découvrit un morceau de papier sur lequel Julie avait écrit : «Je t'attends ce soir à 8h, sous le gros arbre, à l'entrée de la forêt ».

7) Tom avait un peu peur parce qu'il lui était interdit d'aller dans la forêt, surtout la nuit.

8) Mais à $8 \mathrm{~h}$ du soir, il était tout de même au rendez-vous, Julie l'attendait déjà.

9) Sans dire un mot, la petite fille prit la main de Tom et frappa 3 fois sur le tronc du gros arbre.

10) Au bout de quelques minutes, les enfants entendirent un grincement. L'arbre était en train de tourner sur lui-même.

11) Tout à coup, le tronc s'ouvrit et les enfants furent éblouis par la lumière qui inondait l'intérieur de l'arbre. Ils firent quelques pas et l'arbre se referma derrière eux

12) Tom et Julie se trouvaient dans un jardin merveilleux où les fleurs semblaient se parler en chantant. Alors Julie dit à Tom : «Viens, traversons le jardin, il y a une grande fête pour toi, ce soir. Jusqu'à minuit, tu as le droit de demander à notre Roi tout ce que tu veux ».

13) Tom a répondu : «Je veux apprendre à parler avec les oiseaux qui savent tout ce qui se passe dans le ciel, avec les poissons qui savent tout ce qui se passe dans l'eau et avec les fourmis qui savent tout ce qui se passe sur la terre».

14) Et depuis ce jour, Tom est devenu un enfant extrêmement savant. 


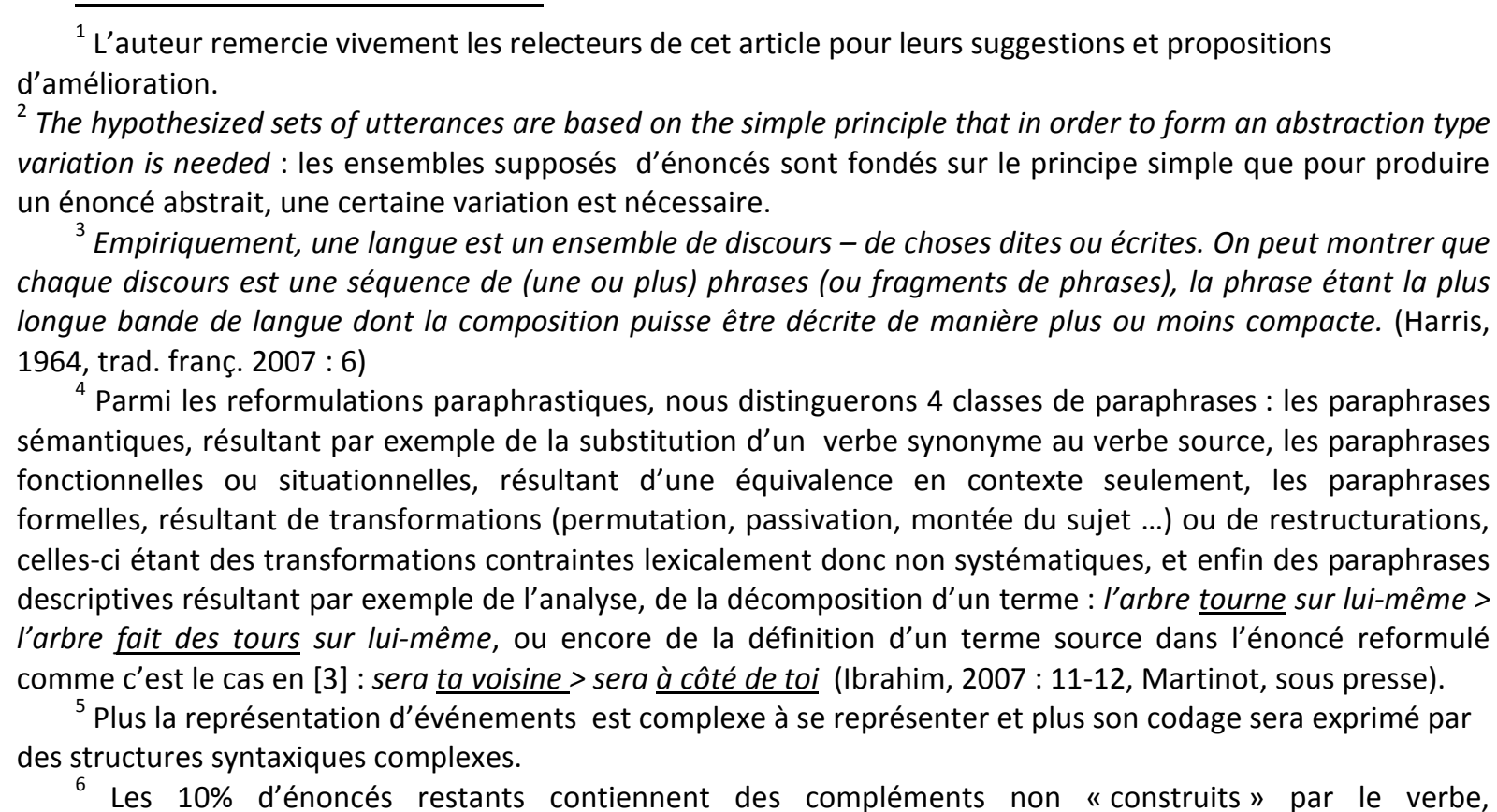
essentiellement des temporels et des locatifs. Ex. Luc a dormi pendant six heures (non construit) VS Les tirs ont duré pendant six heures (construit) (Gross, $1994: 9$ )

${ }^{7}$ La hiérarchie que nous établissons entre la reformulation répétitive (moins complexe) et la reformulation paraphrastique (plus complexe) n'invalide pas l'observation que la répétition d'un énoncé complexe est déjà complexe pour un petit enfant ainsi que le rappelle Tomasello (2003: 175) : un enfant de 1 ans ne peut pas répéter un énoncé contenant une relative.

${ }^{8}$ Dans nos publications de 2000 à 2004, le texte source, lu aux enfants, est un conte d'Europe du Nord ( $L a$ pauvre grenouille, que nous avons rebaptisé pour les publications de 2003 et suivantes en Deux amis malheureux). Le texte source utilisé par la suite, dans le cadre du projet Acquisition et Reformulation coordonné par l'auteur de ces lignes et portant sur 7 langues maternelles différentes, a été rédigé par nos soins (Tom et Julie) et ne contient, dans la mesure du possible, que des éléments d'information accessibles à tout enfant.

${ }^{9}$ L'écart entre l'enfant le plus jeune et le plus âgé de chaque tranche d'âge ne dépasse jamais 3 mois, les enfants avaient donc au moment des enregistrements de 4;0 à 4;3, de 6;0 à 6;3 etc.

10 Les MAD présentent en fait un degré de décomposition et d'explicitation de toutes les relations grammaticales beaucoup plus avancé (Ibrahim, 2010). Nous fournissons ici le premier degré de décomposition qui correspond uniquement à celle du verbe.

${ }^{11}$ Le verbe trouver a une fréquence d'emploi plus élevée que celle du verbe découvrir, ce qui rendrait trouver plus accessible et donc plus facile à employer que découvrir.

${ }^{12}$ Pour certains verbes comme le verbe découvrir, la différence entre les 4-6 ans et les 8-10 ans n'est que quantitative. La reformulation de découvrir par trouver est attestée chez un enfant de 4 ans et 2 de 6 ans.

${ }^{13}$ La MAD de souffler est : dire quelque chose à voix basse de façon à n'être entendu que du destinataire.

14. Ces verbes élémentaires ne sont pas nécessairement des verbes supports (Gross M, 1981, Gross G., 2010, Ibrahim, 1996, Martinot, 1996, 1997), ni systématiquement des light verbs, qui n'ont pas fait l'objet d'une caractérisation syntaxique comme c'est le cas des verbes supports et dont la désignation serait peu cohérente avec notre cadre théorique.

${ }^{15}$ La MAD de il lui a tendu la boîte serait : il a tendu le bras de façon à lui donner la boîte. La MAD de Tom souleva le couvercle (de la boîte) serait : il a saisi le couvercle en le déplaçant lentement vers le haut de façon à ouvrir complètement la boîte.

${ }^{16}$ On peut donner beaucoup de choses mais on n'offre que des cadeaux, ce qui est le cas ici.

${ }^{17}$ Les reformulations que nous avons qualifiées jusqu'à présent de transformations et de restructurations ne le sont jamais de façon stricte comme ce serait le cas dans un travail de description des propriétés transformationnelles d'un ensemble de phrases. Les reformulations sont rarement «pures », l'étiquette donnée à la procédure de reformulation correspond à la modification la plus importante.

${ }^{18}$ Le taux de répétition ne peut être utilisé comme moyen de mesure du fait que les enfants étaient invités à raconter l'histoire " avec leurs mots à eux ", les quelques répétitions sont donc produites " malgré eux "; 
quant aux reformulations qui changent le sens, elles ne se prêtent pas à une mesure relative : on ne peut pas dire que telle prédication est plus ou moins modifiée que telle autre, ni que telle modification est plus ou moins complexe à produire, enfin, comme pour la répétition, on peut supposer que les changements de sens sont produits malgré les enfants.

${ }^{19} 30$ enfants sur 60 l'ont paraphrasée, et donc le nombre d'enfants qui l'ont reformulée est encore plus grand.

${ }^{20}$ Cette conclusion doit cependant être nuancée: les restructurations dépendent des constructions, disponibles dans chaque langue, de tel mot lexical. Si un verbe ou un nom ne peuvent être restructurés, $c^{\prime}$ est par exemple le cas de l'équivalent italien teneva per mano una bambina (Martinot \& Gerolimich, sous presse), alors d'autres procédures de reformulation paraphrastique seront prises en compte pour mesurer le degré de complexification attesté dans la reformulation.

${ }^{21}$ La séquence source reprend trois fois de suite la même construction, d'abord avec les oiseaux qui savent tout ce qui se passe dans le ciel puis avec les poissons qui savent (...) dans l'eau et enfin avec les fourmis qui savent (...) sur la terre. Nous retenons la construction la mieux réussie chez chaque enfant, donc selon les cas, il s'agira des oiseaux, des poissons ou des fourmis.

${ }^{22}$ Nous analysons tout ce qui se passe dans le ciel comme une complétive de savent plutôt que comme une relative ayant pour antécédent (tout) ce du fait que (tout) ce ne fonctionne pas comme un véritable antécédent, comme ce serait le cas avec un nom, mais fait partie de la suite tout ce qui se passe ... (cf. *qui savent quoi se passe, die wissen, was passiert, who know, what happens)

${ }^{23}$ E note un élément effacé. 